\title{
Ductus: A software package for the study of handwriting production
}

\author{
ERIC GUINET \\ LPNC, CNRS UMR 5105, Université Pierre Mendès France, Grenoble, France \\ AND \\ SONIA KandeL \\ LPNC, CNRS UMR 5105, Université Pierre Mendès France, Grenoble, France \\ and Institut Universitaire de France, France
}

\begin{abstract}
Ductus is a software tool designed to analyze and aid understanding of the processes underlying handwriting production. Ductus is a digitizer-based device that provides online information on the handwriting process. It consists of two distinct modules that operate independently. The first module concerns stimulus presentation. It is particularly suited to experiments with children and patients presenting handwriting pathologies. The second module is devoted to data analysis. Apart from the geometrical aspects of handwriting, such as trajectory formation, Ductus provides a wide range of kinematic information, such as velocity, duration, fluency, and pauses, linked to the mastery of the movement itself. Ductus is available free from the authors. It works on a Windows platform with Wacom digitizers.
\end{abstract}

Writing is a psychomotor activity that involves a series of high- and low-level processes (e.g., Berninger \& Graham, 1998; Hayes, 1996; Van Galen, 1991). The former essentially refer to the compositional aspects of writing, whereas the latter are more related to the transcription skills that are involved in the handwriting process (cf. Graham \& Harris, 2000). According to Van Galen's model, handwriting production is the result of a series of processing stages organized in a hierarchical manner. The higher order processing levels - activation of intentions, semantic retrieval, syntactic construction - deal with the more abstract aspects of linguistic production and are common to the production of other linguistic tasks such as speech (Levelt, 1989). Writing and speech processes differ at the spelling module, which stores the orthographic representations that code information on the spelling of words and is at the interface between the high- and lowlevel modules. The low-level modules that Van Galen called "motor modules" are involved in processing allograph selection, size control, and muscular adjustment. Alamargot, Chesnet, Dansac, and Ros (2006) presented a commercial software package called Eye and Pen that is particularly well adapted to the study of the compositional and higher order processes of writing. As a complement to that system, in this article, we present Ductus, a free software package for the study of the lower order processes involved in handwriting production. Besides the geometrical aspects of handwriting like trajectory formation, Ductus provides a wide range of analytic information linked to the proficiency of the movement itself: velocity, duration, fluency, and pauses. These measures are particularly well suited to provide insight into handwriting acquisition and its pathology. Indeed, these analytical measures provide evidence on handwriting processing that global-holistic methods cannot detect (see Rosenblum, Weiss, \& Parush, 2004 , for a review on this issue).

At least $50 \%$ of a child's school day is spent in writing tasks, and some of them have to be executed as quickly as possible (Amundson \& Weil, 1996; McHale \& Cermak, 1992; Tseng \& Chow, 2000). The mastery of handwriting is therefore essential to achieving a satisfactory academic performance. Moreover, teachers assign higher grades to neatly written papers than to poorly handwritten papers presenting the same content (e.g., Chase, 1986). There are few studies quantifying the proportion of children with handwriting difficulties. Up to one third of schoolchildren have poor handwriting (Rosenblum et al., 2004; Rubin \& Henderson, 1982; Smits-Engelsman, Van Galen, \& Michels, 1995). Furthermore, $30 \%-40 \%$ of the children with learning disabilities seem to have handwriting difficulties (Cratty, 1994). It is, therefore, essential to detect handwriting difficulties early, so that the children can benefit from therapy as soon as possible and overcome difficulties with the mechanical aspects of handwriting, or learn to cope with them (Phelps \& Stempel, 1988). Most scales evaluate handwriting quality on the basis of "legibility." They are global-holistic scales based on the geometrical aspects of handwriting. Most handwriting quality scales are based

S. Kandel, sonia.kandel@upmf-grenoble.fr 
on the product but not on the process of handwriting (see Rosenblum et al., 2004, for a review). However, the cause of a poor product is the lack of mastery in the production process. Ductus is a digitizer-based software that provides tools for the online study of the handwriting process. It facilitates the extraction of several characteristics of handwriting processing, such as movement duration, velocity, duration, fluency, and pauses.

Some studies have used analytic measures on handwriting gestures to gain an understanding of the difficulties of handwriting acquisition at school. Rosenblum, Parush, and Weiss (2003b) used information on pen pressure to analyze a series of kinematic variables in play when the child has his/her hand in the air while writing. This kind of data reveals information on what the participant does with his/her hand when he/she is not writing but planning or preparing the next movement sequence. The authors showed that the "in air" time for poor writers is significantly longer than for proficient writers. This finding, which they called the in air phenomenon, is extremely useful for detecting children with handwriting problems.

Ductus is also particularly useful for investigating the relationship between spelling and writing proficiency. Graham, Harris, and Chorzempa (2002) noted that spelling imposes strong attentional and mnemonic demands, especially on young children, that affect other aspects of the writing process (Berninger, 1999; Graham, 1999). The analytical study of handwriting production revealed, for instance, how the information encoded by the orthographic representations at the spelling level regulates motor outputs in children (Kandel, Soler, Valdois, \& Gros, 2006; Kandel \& Valdois, 2006a, 2006b) and adults (Kandel, Alvarez, \& Vallée, 2006, 2008). The authors showed that sublexical units regulate motor programming in handwriting, thereby producing significant movement duration and/or dysfluency increases at specific locations within a word. These increases appear at syllable boundaries - for example, between $o$ and $m$ in the word fromage ("cheese") - as well as at morpheme boundaries - in the word montage ("putting up"), for instance, between the root mont and the suffix age. The temporal increases are due to the simultaneous processing of the syllabic and/or morphological components of the word and local parameters, such as rotation direction, letter size, and force, necessary to execute the current movement sequence (cf. Van Galen, 1991). In first grade - that is, at the beginning of the acquisition process - the handwriting process is also regulated by graphophonological constraints. When a six-year-old child has to write the word chanson ([Sãsõ]; "song"), for example, he/she segments the initial syllable into graphemes. He/she programs the first grapheme $c h[\mathrm{~S}]$ before movement initiation, then prepares the second grapheme an [ã] in parallel with local parameters (direction, size, and force), and finally son [sõ] as a whole syllable unit. To summarize, several studies using online measures of the handwriting movement reveal that the orthographic representations used in writing production present a linguistic format. Letters, graphemes, syllables, and mor- phemes modulate the processes involved in the production of handwriting movements. The way these linguistic units interact during the writing process depends on the orthographic characteristics of each language (Kandel \& Valdois, 2006a).

These kinds of studies require stimulus presentation software that optimizes the acquisition of handwriting data. Researchers also need efficient data analysis software that facilitates the segmentation of the trajectories and velocity profiles. This analysis is very time-consuming and is subject to errors when done by hand. There are other software packages that have some of the functions Ductus provides. The information on these packages is quite scarce and there is no detailed information on their functionalities. The software developed by Mai and Marquardt (1992) is particularly efficient for the automatic segmentation of strokes (i.e., a movement sequence executed between two absolute velocity minima), but requires other tools, developed in MATLAB for example, for optimal use. It is also a commercial product, which may limit its use for some researchers. OASIS (De Jong, Hulstijn, Kosterman, \& Smits-Engelsman, 1996) is similar to Ductus but is not freely available. Finally, ComPET, the evolution of POET, is also similar to Ductus, but requires further programming (in MATLAB) for complete efficiency (Rosenblum, Parush, \& Weiss, 2003a). In the latter, only the data acquisition module seems to be available. The users have to send the data files to the authors for data analysis. SpellWrite is a free software package in which online processes can be studied, but the number of measures it allows the experimenter to implement is rather limited (see Álvarez, Cottrell, \& Afonso, 2009, for an example of the kind of information it can provide). For all these reasons, Ductus seems to be a good alternative, because it is ready to use in its present state, it can perform a wide range of measurements, and access to it is free with a simple request to the authors.

Ductus integrates stimulus presentation and movement analysis into one software package. Data acquisition is conducted with a module that acquires data on the handwriting movement from a digitizer. It was designed to adapt to different experimental possibilities and acquire the data in a very "ecological" fashion. It is adapted for studying adults' and children's handwriting production. Thus, when using Ductus, the children can have the feeling of writing, as they usually do in their school tasks. The function of the other module is to visualize and analyze the recorded data. A great number of measurements can be made automatically. Both the data acquisition and kinematic analysis "modules" are simple to use, so they can be used by nonmotor control researchers. The two modules function on Windows platforms (2000, XP, Vista) with Wacom digitizers. Ductus can be used in French and English.

\section{Stimulus Presentation Module}

The data acquisition module can be used with any model or size of a Wacom digitizer. The handwritten data can be recorded from different kinds of stimuli. The stimulus presentation can be visual - a word (.txt file format), text 


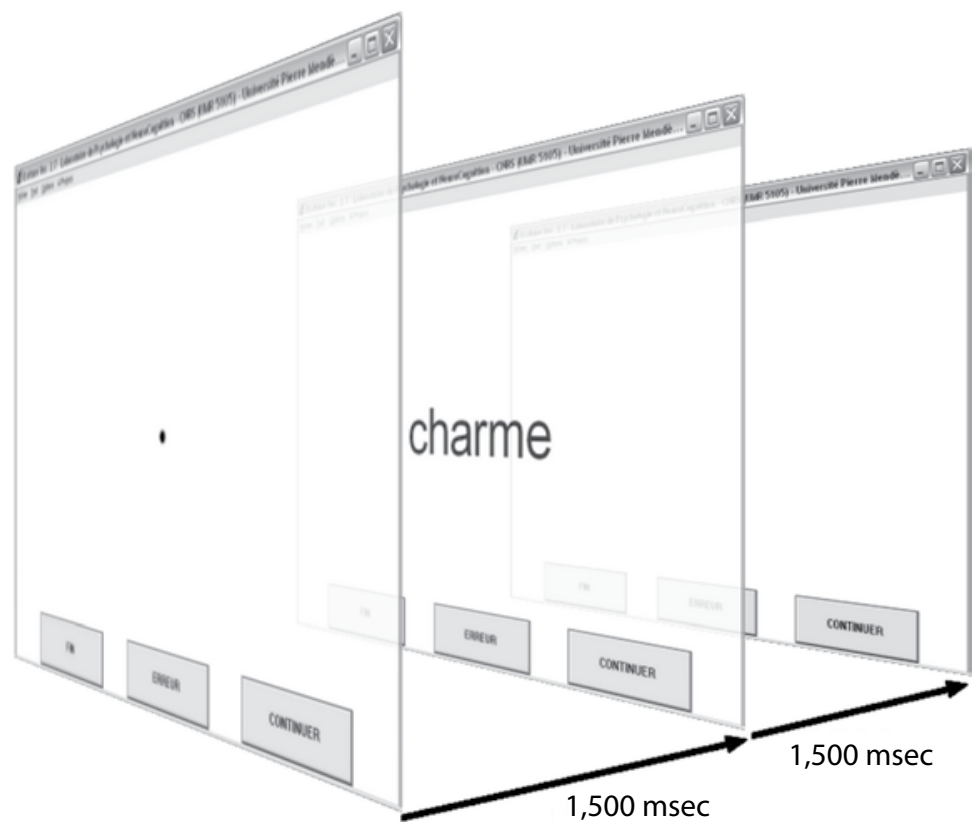

Figure 1. Example of stimulus presentation in the data acquisition module.

(.bmp file format), or picture (.bmp file format) —as well as auditory (.wav file format). Two presentation modes may be combined within an experimental set. Figure 1 presents an example of stimulus presentation in the data acquisition module. The experiment begins with an auditory signal (beep) informing the participant that a stimulus will be presented; then, a fixation point at the center of the screen indicates the location where the stimulus will appear. The presentation time of the fixation point can be manipulated by the experimenter (in milliseconds); then, the visual stimulus replaces the fixation point.

In Kandel, Herault, Grosjacques, Lambert, and Fayol (2009), for instance, third, fourth, and fifth graders wrote words according to the procedure presented in Figure 1. In this experiment, the child's task was to write the target word on lined paper with an ink pen (Wacom Intuos Inking Pen) on the digitizer. The paper was the same as the one French children usually write on at school. This places the child in a rather "ecological" writing situation. Once he/she finished writing the word, the experimenter clicked on the "Continuer" (Continue) button located at the bottom right of the screen to present the next stimulus. If there is a mistake or the child wishes to write the word again, the experimenter may click on the "Erreur" (Error) button located at the bottom center of the screen and restart the whole procedure. When this is done, the same word is presented and the child writes it a second time. The stimulus presentation module records the "mistaken" and the "nonmistaken" productions so both traces can be analyzed according to the purposes of the experiment. The stimulus presentation stops automatically when all the words in the stimulus file have been presented. A small dialog box that appears at the center of the screen informs the experimenter that all the stimuli in the file have been presented. If the experimenter needs to end the stimulus presentation module before the end of the stimulus file, he/she must click on the button "Fin" (End).

The handwriting data are recorded at a sampling frequency of $200 \mathrm{~Hz}$. The sampling frequency depends on the digitizer's capacities and not on the data acquisition module. The recorded information is the following:

1. Pen position on the digitizer surface ( $x, y$ raw coordinates) - that is, when the pen is in contact with the paper. It is also possible to record the pen position on the digitizer area even if there is no contact between the pen and the digitizer - that is, when the pen is in the air. The availability of this data depends on the digitizer's capacities. For a Wacom Intuos 3 (A5 format), the pen is detected up to $6 \mathrm{~mm}$ above the digitizer's surface.

2. Pen pressure refers to the pressure of the pen on the digitizer's surface. It is received in nonscaled units rather than in grams per area.

3. Latency (msec) refers to the time between the beginning of stimulus presentation and the instant at which the pen touches the surface of the digitizer.

4. Event landmarks is an option that allows the experimenter to put a "mark" at any time on what the participant produces. If there is a landmark, Ductus scores the event as 1; if there is no landmark, it scores the event as 0. Kandel and Valdois (2006a, 2006b), for example, used this kind of "mark" in a copying task to indicate the moment at which a child raised his/her eyes to find more information on the spelling of the word he/she had to write.

The data are the raw $x, y$, and pressure coordinates provided by the digitizer and the event landmarks. The stimulus presentation module records all this information in a .txt file. The data analysis module reads and performs calculations on this data. 

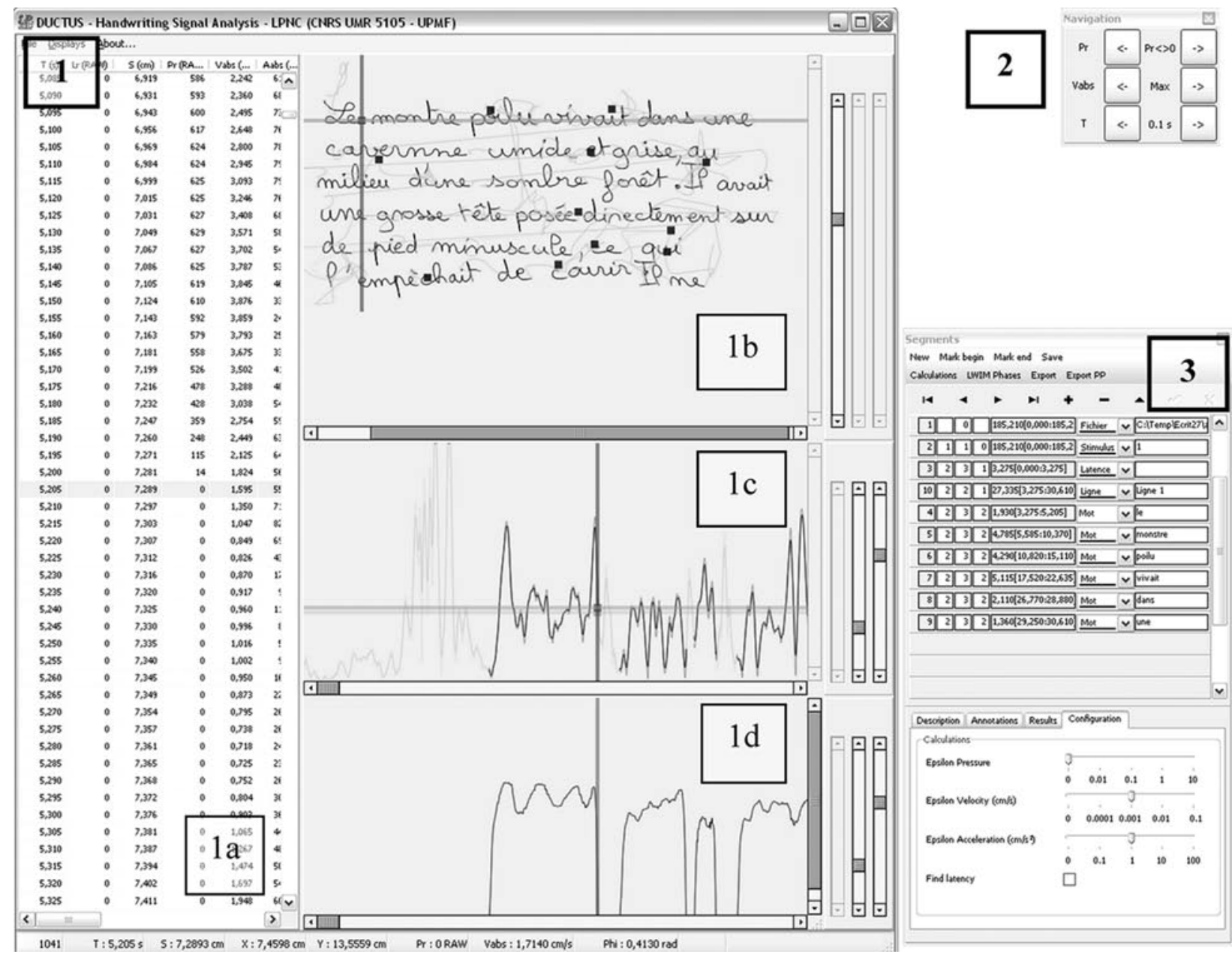

Figure 2. Data analysis interface. Part 1 concerns data visualization; 1 a is the data table with $x$ and $y$ values, absolute velocity, and acceleration, as well as pressure; $1 \mathrm{~b}$ is the movement trajectory; $1 \mathrm{c}$ is the velocity profile; and $1 \mathrm{~d}$ is the pressure profile. Part 2 facilitates navigation in any of the Part 1 areas according to pressure, velocity, and temporal criteria. Part 3 is designed to segment the information and perform calculations on the selected segment.

\section{Data Analysis}

Basic calculations. The trajectory raw data $(x y)$ are converted to centimeters and are then smoothed with a lowband Butterworth first-order filter with a $12-\mathrm{Hz}$ cutoff frequency (Rabiner \& Gold, 1975). The filter is applied twice. The first time it is applied in the usual chronological way. The second time it is applied in a reversed chronological procedure to limit the temporal gap caused by Butterworth's filter between the raw and filtered data. With this data, Ductus calculates the kinematic and geometrical parameters of the handwriting movement: horizontal, vertical, and absolute velocities of the pen tip (expressed in $\mathrm{cm} / \mathrm{sec}$ ); horizontal, vertical, and absolute accelerations $\left(\mathrm{cm} / \mathrm{sec}^{2}\right)$; trajectory direction or angle with the horizontal line (in radians); the variation of this angle with respect to time (angular velocity in $\mathrm{rad} / \mathrm{sec}$ ); and the trajectory curvature expressed in $\mathrm{cm}^{-1}$ (Bertrand, 1864). Note that curvature is equal to 0 for a point on a straight trajectory and $\pm \infty$ for a cusp point on a trajectory. Pressure data are filtered with a median filter of 7 sample width. Ductus's graphic interface presents the re- sults of these calculations (Figure 2, Parts 1c and 1d). These data are used for the elaboration of movement parameters such as duration, mean velocity, and the like.

Visualization and navigation interface. Figure 2 presents Ductus's data analysis interface. This graphic interface consists of several windows.

Part 1 concerns data visualization. On the left (Part 1a), there is a table with different kinds of data (time, $x, y$, absolute velocity, absolute acceleration, and pressure). The user can decide which kind of information should be presented in this table by clicking on a dialogue box: (1) the raw $x y$, pressure, and landmark data; (2) the converted $x, y$ coordinates in centimeters; (3) the filtered data; and (4) the calculated data. Part $1 \mathrm{~b}$ shows the movement trajectory. The bold line indicates the part of the writing movement that is produced with pressure $>0$. This is the trajectory of the pen tip that touched the digitizer and that can be seen on the paper if the experiment was conducted with an ink pen. The gray lines show the air movements made when the pen does not touch the digitizer's sur- 
face; that is, the movements that the digitizer detected that were made with pressure 0 . There is a zoom that facilitates the visualization of the parts of the trajectory the user wants to focus on. Part $1 \mathrm{~d}$ is a pressure profile. It is designed to visualize both the pressure distribution as a function of time and the pauses of the writer while producing his/her movement. The user can click on any of the four parts and the cursor will appear in red and green on the others. For example, if the user wants to find the end of an upstroke and the beginning of a downstroke (defined by an absolute velocity minimum), he/she can click on a velocity minimum in Part $1 \mathrm{c}$, and the cursor in Parts $1 \mathrm{a}, 1 \mathrm{~b}$, and $1 \mathrm{~d}$ will automatically appear at the corresponding point.

Part 2 facilitates navigation in any of the Part 1 areas according to three criteria. The first criterion is pressure. The values it uses are pressure $>0$ and pressure $=0$. With one click, the user can pass from a point where pressure $>0$ to the following point where pressure $>0$. These clicks are coordinated with the data table (Part 1a), trajectory (Part 1b), and velocity and pressure profiles (Parts 1c and $1 \mathrm{~d}$, respectively), such that a click will automatically move the cursor to the corresponding point in each window of Part 1. Likewise, it is possible to pass from a given point to the following band, where pressure $=0$, and so on to the following band, where pressure $=0$.

The second navigation criterion is velocity maxima and minima. The user can pass from velocity maxima to velocity maxima or from velocity minima to velocity minima. Again, the cursor will appear in all the Part 1 windows. Finally, the third navigation criterion is related to time. By clicking on the button, the user can move through the four windows of Part 1 by intervals of $0.1 \mathrm{sec}$. It should also be pointed out that the three operations can be done forward and backward throughout the whole production area. Finally, the three kinds of operations can be repeated as many times as the user likes. These criteria are the ones that most researchers use to segment continuous cursive handwriting into discrete units. Using them for navigation significantly decreases the time taken during the segmentation process.

The information provided by Part 3 is distributed in four fields. The "Description" field contains information such as the type of digitizer used and information that concerns the whole production file, such as latency (the time period between stimulus presentation and movement initiation), the number of recorded coordinates, and so on. This field can be modified according to users' needs with a dialog box. In the "Annotation" area, the user can write whatever he/she wishes. The "Configuration" field refers to the parameters that determine how the calculations are going to be conducted. The "Results" field provides different kinds of information calculated on a predefined segment (segment writing and in air duration, writing and in air trajectory, writing and in air dysfluency calculated as the number of velocity maxima), mean writing and in-air velocity, number of in-air periods, mean duration of in-air periods, etc.). Handwriting is usually a continuous trajectory, and to gain understanding of handwriting production, researchers need to segment this trajectory into smaller units such as letters or syllables. Part 3 is designed to select segments and perform calculations on them.

The user determines the size of the segment. The selected segments can be organized in a hierarchical fashion (Figure 3). A text can be segmented into paragraphs and the latter into sentences. Kandel and colleagues, for example, segmented words into syllables (Kandel \& Valdois, 2006b) and the syllables in turn were segmented into graphemes and/or letters (Kandel, Soler, et al., 2006). The hierarchical structure of these segments can be saved in an XML format.

The segment is defined by clicking on "New." This click indicates that a new segment needs to be created. Then, the user has to click on "Mark begin" to indicate the beginning of the segment and "Mark end" to indicate the end of the segment. The user can indicate the kind of segment that has been created (syllable, letter, etc.). Once the trajectory is segmented, the user clicks on "Calculations" to obtain the desired values (duration, mean velocity, etc.) for each segment. This information is obtained by calculations on the raw data and is presented in the "Results" field. It concerns different aspects related to what the user wishes to study. A writing phase, for example, can be characterized by a movement trajectory that is done with pressure, velocity, and acceleration $>0$. An "in air" phase, as stud-

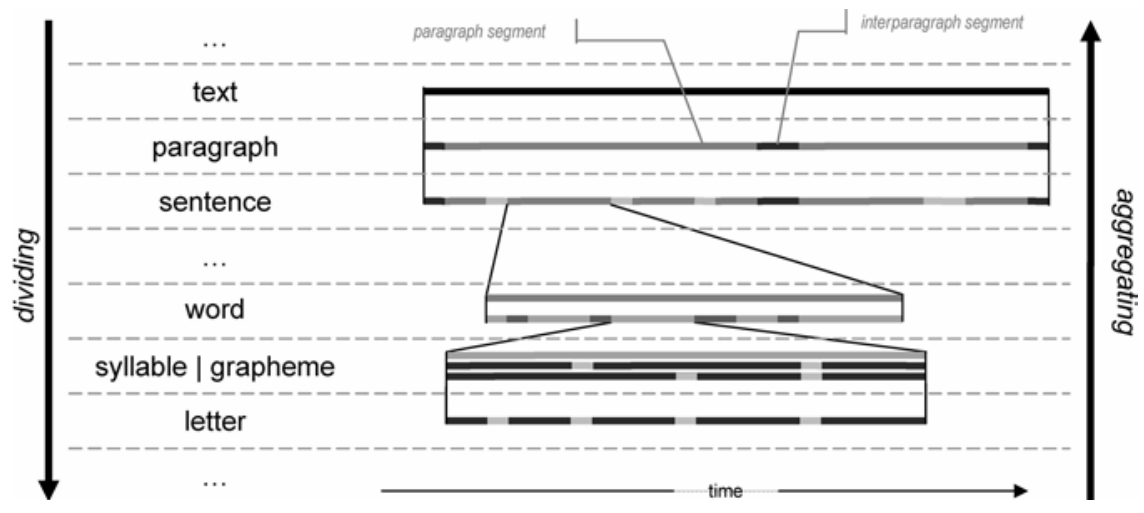

Figure 3. The possible segmentation levels of a trajectory. 
Table 1

Calculations Conducted on the Segmentation of the Word "les" Into Letters and Strokes

\begin{tabular}{|c|c|c|c|c|c|c|c|c|c|}
\hline & & & & \multirow{4}{*}{$\begin{array}{c}\text { Trajectory } \\
\text { Total } \\
(\mathrm{cm})\end{array}$} & \multicolumn{5}{|c|}{ Velocity } \\
\hline & \multirow{2}{*}{\multicolumn{3}{|c|}{ Duration (sec) }} & & \multirow{3}{*}{$\begin{array}{c}M \\
(\mathrm{~cm} / \mathrm{sec}) \\
\end{array}$} & \multicolumn{2}{|c|}{ Maximum } & \multicolumn{2}{|c|}{ Minimum } \\
\hline & & & & & & & & & \\
\hline & Begin & End & Total & & & No. & $(\mathrm{cm} / \mathrm{sec})$ & No. & $(\mathrm{cm} / \mathrm{sec})$ \\
\hline Word: les & 1.020 & 2.750 & 1.730 & 12.86 & 7.43 & 8 & 10.28 & 9 & 4.08 \\
\hline Letter: 1 & 1.020 & 1.625 & 0.605 & 5.96 & 9.85 & 2 & 16.23 & 3 & 4.26 \\
\hline Stroke: 0-1 & 1.020 & 1.335 & 0.315 & 3.04 & 9.65 & 1 & 14.90 & 2 & 3.61 \\
\hline Stroke: 1-2 & 1.335 & 1.625 & 0.290 & 2.92 & 10.07 & 1 & 17.56 & 2 & 5.14 \\
\hline Letter: e & 1.625 & 2.000 & 0.375 & 3.14 & 8.37 & 2 & 10.75 & 2 & 6.00 \\
\hline Stroke: $\mathbf{2}-\mathbf{3}$ & 1.625 & 1.840 & 0.215 & 1.89 & 8.79 & 1 & 11.53 & 1 & 4.84 \\
\hline Stroke: $3-4$ & 1.840 & 2.000 & 0.160 & 1.25 & 7.80 & 1 & 9.98 & 2 & 6.00 \\
\hline Letter: $\mathbf{s}$ & 2.000 & 2.750 & 0.750 & 3.76 & 5.01 & 4 & 7.08 & 5 & 3.82 \\
\hline Stroke: $\mathbf{4 - 5}$ & 2.000 & 2.320 & 0.320 & 2.03 & 6.34 & 1 & 11.22 & 2 & 3.64 \\
\hline Stroke: 5-6 & 2.320 & 2.750 & 0.430 & 1.73 & 4.03 & 3 & 5.70 & 4 & 2.99 \\
\hline
\end{tabular}

ied by Rosenblum et al. (2003b), for instance, corresponds to a pen trajectory that does not touch the digitizer's surface (i.e., pressure $=0$ ). A stable phase called "motionless" that we sometimes observe in dysgraphic children's productions concerns a temporal period in which the pen tip touches the digitizer's surface but does not move (i.e., pressure $>0$, but velocity and acceleration $=0$ ). The user can choose parameters such as mean velocity or movement duration by clicking on the dialog box.

Segmentation example. Figure 4 presents the example of how the trajectory of a word "les" (trajectory going from point 0 to 6 ) is segmented into letters (points $0-2$, $2-4,4-6)$ and in turn into strokes (points 0-1 upstroke and 1-2 downstroke, 2-3 upstroke and 3-4 downstroke, and $4-5$ upstroke and 5-6 downstroke). In this example we
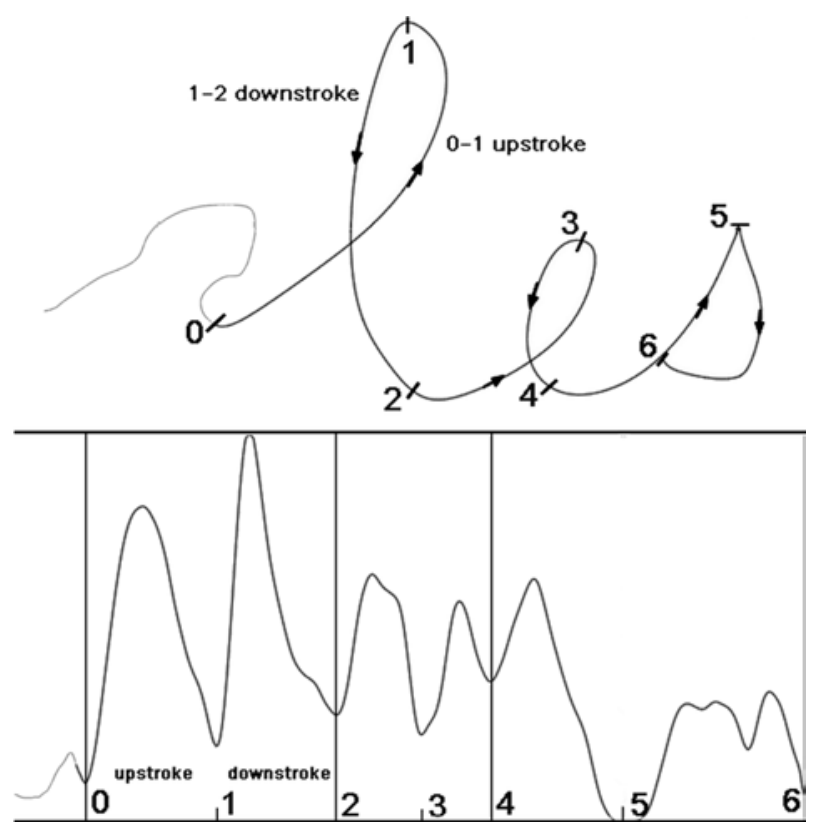

Figure 4. Trajectory and velocity profile of the word "les." Segmentation example of upstroke and downstroke. have three hierarchical levels: word, letter, and stroke. The calculations were conducted on each level. Table 1 shows the results. We observe that the word "les" was written in $1.73 \mathrm{sec}$ at a mean tangential velocity of $7.43 \mathrm{~cm} / \mathrm{sec}$. Mean velocity decreased from the beginning to the end of the word: 9.85 for "l," 8.37 for "e," and 5.01 for "s." Another observation is the fact that the word's last stroke (the downstroke of the "s," points 5-6) was the most dysfluent, with 3 velocity maxima versus 1 and 2 for the rest of the strokes.

Data and result export. With Ductus, the data can be exported in two ways. The first way is to perform a cut/paste procedure from the calculation information provided by Ductus to a spreadsheet program. This was how Table 1 was done. The second way is to save the information in a .txt format file. It will record the information on the occurrence that the user selected as well as the samples that correspond to this segment.

Software validation. We produced data files with various types of figures, letters, and words, and we verified that Ductus's calculations retrieved the correct geometrical parameters of the trajectories (e.g., perimeter and radius of curvature). This can be verified by using an ink pen (Intuos Inking Pen for Wacom digitizers) to produce a figure stuck to the digitizer, and by then measuring the geometrical parameters on the paper. To control for the temporal aspects of data acquisition, we filmed some of the participants' productions, and made sure that the information provided by Ductus (e.g., word duration) was in agreement with the measurements provided by the camera. A supplementary verification was done by generating data files with perfect circles and spirals with specific radius of curvature.

Furthermore, the data acquired and analyzed with Ductus is consistent with data collected in other experiments conducted with previous software. For example, the developmental results published by Kandel et al. (2009), using Ductus, reproduced the syllable-by-syllable writing pattern observed in children by Kandel and Valdois (2006a, 2006b) and Kandel, Soler, et al. (2006), suggesting that French children use syllables as processing units to write words. 


\section{Conclusion}

Ductus is a new software that will facilitate the study of handwriting production in children and adults. Furthermore, it is particularly well adapted to the investigation of handwriting pathologies. The software is free. Any researchers interested in it can ask the corresponding author to send it.

\section{AUTHOR NOTE}

This research was supported by a grant from the Région RhôneAlpes (Cluster Handicap, Viellissement et Neurosciences) to S.K. We are grateful to Muriel Lobier for linguistic support. Correspondence concerning this article should be addressed to S. Kandel, CNRS UMR 5105, Université Pierre Mendès France, Institut Universitaire de France, Laboratoire de Psychologie et Neurocognition, B.P. 47-BSHM, 38040 Grenoble Cedex 09, France (e-mail: sonia.kandel@upmf-grenoble.fr).

\section{REFERENCES}

Alamargot, D., Chesnet, D., Dansac, C., \& Ros, C. (2006). Eye and Pen: A new device for studying reading during writing. Behavior Research Methods, 38, 287-299.

Álvarez, C. J., Cottrell, D., \& Afonso, O. (2009). Writing dictated words and picture names: Syllabic boundaries affect execution in Spanish. Applied Psycholinguistics, 30, 205-223.

Amundson, S. J., \& WeIL, M. (1996). Prewriting and handwriting skills. In J. Case-Smith, A. S. Allen, \& P. N. Pratt (Eds.), Occupational therapy for children (pp. 524-541). St. Louis: Mosby-Year Book.

BERNINGER, V. (1999). Coordinating transcription and text generation in working memory during composing: Automatic and constructive processes. Learning Disability Quarterly, 22, 99-112.

BERNINGER, V., \& GraHAM, S. (1998). Language by hand: A synthesis of a decade of research in handwriting. Handwriting Review, 12, 11-25.

Bertrand, J. (1864). Traité de calcul différentiel et de calcul intégral. Paris: Gauthier-Villars.

CHAsE, C. (1986). Essay test scoring: Interaction of relevant variables. Journal of Educational Measurement, 23, 33-41.

CRATTY, B. J. (1994). Clumsy child syndromes, descriptions, evaluation and remediation. Langhorne, PA: Harwood Academic.

De Jong, W. P., Hulstijn, W., Kosterman, B. J. M., \& SmitsENGELSMAN, B. C. M. (1996). OASIS software and its application in experimental handwriting research. In M. L. Simner, C. G. Leedham, \& A. J. W. M. Thomassen (Eds.), Handwriting and drawing research: Basic and applied issues (pp. 429-440). Amsterdam: IOS Press.

Graham, S. (1999). Handwriting and spelling instruction for students with learning disabilities: A review. Learning Disability Quarterly, 22, 78-98

Graham, S., \& Harris, K. (2000). The role of self-regulation and transcription skills in writing and writing development. Educational Psychologist, 35, 3-12.

Graham, S., Harris, K., \& Chorzempa, B. F. (2002). Contribution of spelling instruction to the spelling, writing and reading of poor spellers. Journal of Educational Psychology, 94, 669-686.

HAYES, J. R. (1996). A new framework for understanding cognition and affect in writing. In C. M. Levy \& S. Ransdell (Eds.), The science of writing: Theories, methods, individual differences, and applications (pp. 1-26). Mahwah, NJ: Erlbaum.
Kandel, S., Alvarez, C., \& VAllée, N. (2006). Syllables as processing units in handwriting production. Journal of Experimental Psychology: Human Perception \& Performance, 32, 18-31.

Kandel, S., Alvarez, C., \& Vallée, N. (2008). Morphemes also serve as processing units in handwriting production. In M. Baciu (Ed.), Neuropsychology and cognition of language behavioural, neuropsychological and neuroimaging studies of spoken and written language. Kerala, India: Research Signpost.

Kandel, S., Herault, L., Grosjacques, G., Lambert, E., \& Fayol, M. (2009). Orthographic vs. phonologic syllables in handwriting production. Cognition, 110, 440-444

KANDEl, S., Soler, O., VAldoIs, S., \& Gros, C. (2006). Graphemes as motor units in the acquisition of writing skills. Reading \& Writing, 19, 313-337.

Kandel, S., \& Valdois, S. (2006a). French and Spanish-speaking children use different visual and motor units during spelling acquisition. Language \& Cognitive Processes, 21, 531-561.

KANDEL, S., \& VALDOIS, S. (2006b). Syllables as functional units in a copying task. Language \& Cognitive Processes, 21, 432-452.

LeVelt, W. J. M. (1989). Speaking: From intention to articulation. Cambridge, MA: MIT Press.

MAI, N., \& MARQUARDT, C. (1992). CS Computer-Assisted Movement Analysis in Handwriting. Operational manual. Munich: MedCom Verlag.

McHale, K., \& Cermak, S. A. (1992). Fine motor activities in elementary school: Preliminary findings and provisional implications for children with fine motor problems. American Journal of Occupational Therapy, 46, 898-892.

Phelps, J., \& STEMPEL, L. (1988). The children's handwriting evaluation scale for manuscript writing. Reading Improvement, 25, 247-254.

RABINER, L. R., \& GolD, B. (1975). Theory and application of digital signal processing. Englewood Cliffs, NJ: Prentice Hall.

Rosenblum, S., Parush, S., \& Weiss, P. L. (2003a). Computerized temporal handwriting characteristics of proficient and poor handwriters American Journal of Occupational Therapy, 57, 129-138.

Rosenblum, S., Parush, S., \& Weiss, P. L. (2003b). The in air phenomenon: Temporal and spatial correlates of the handwriting process. Perceptual \& Motor Skills, 96, 933-954.

Rosenblum, S., Weiss, P. L., \& Parush, S. (2004). Handwriting evaluation for developmental dysgraphia: Process versus product. Reading \& Writing, 17, 433-458.

Rubin, N., \& Henderson, S. E. (1982). Two sides of the same coin: Variation in 33 teaching methods and failure to learn to write. Special Education: Forward Trends, 9, 17-24.

Smits-Engelsman, B. C. M., Van Galen, G. P., \& Michels, C. G. J. (1995). Teachers' assessment of the motor proficiency level and motor writing skills of elementary school pupils. In B. C. M. SmitsEngelsman, Theory-based diagnosis of fine-motor coordination development and deficiencies using handwriting tasks. Unpublished doctoral dissertation, University of Nijmegen.

TsenG, M. H., \& ChOw, S. M. K. (2000). Perceptual-motor function of school-age children with slow handwriting speed. American Journal of Occupational Therapy, 54, 83-88.

VAN GALEN, G. P. (1991). Handwriting: Issues for a psychomotor theory. Human Movement Science, 10, 165-191.

(Manuscript received April 17, 2009; revision accepted for publication September 6, 2009.) 